\title{
Compound PDE-Based Additive Denoising Solution Combining an Improved Anisotropic Diffusion Model to a 2D Gaussian Filter Kernel
}

\author{
Tudor Barbu' ${ }^{1, *}$ and C. Moroşanu ${ }^{2}$ \\ ${ }^{1}$ Institute of Computer Science of the Romanian Academy, Iasi, Romania. \\ ${ }^{2}$ Department of Mathematics, Al.I. Cuza University of Iasi, Iasi, Romania.
}

Received 27 March 2018; Accepted (in revised version) 26 May 2018.

\begin{abstract}
A second-order nonlinear anisotropic diffusion-based model for Gaussian additive noise removal is proposed. The method is based on a properly constructed edgestopping function and provides an efficient detail-preserving denoising. It removes additive noise, overcomes blurring effect, reduces the image staircasing and does not generate multiplicative noise, thus preserving boundaries and all the essential image features very well. The corresponding PDE model is solved by a robust finite-difference based iterative scheme consistent with the diffusion model. The method converges very fast to the model solution, the existence and regularity of which is rigorously proved.
\end{abstract}

AMS subject classifications: 35Bxx, 94A08, 35K55, 35K60, 35Qxx, 65Nxx

Key words: Image restoration, nonlinear anisotropic diffusion, qualitative properties of solutions, boundary value problems for nonlinear parabolic PDE, Leray-Schauder principle.

\section{Introduction}

Image denoising is one of fundamental tasks in image processing. However, the classic $2 D$ image filters often produce undesired blurring, which affects the edges and other essential image details [10], so that a feature-preserving restoration still represents a serious challenge.

The nonlinear partial differential equations (PDEs) have been increasingly used in the image denoising and restoration in the last three decades. They provide a good solution to the problem since Perona and Malik [18] introduced their celebrated anisotropic diffusion algorithm. Since then, various nonlinear second-order diffusion-based restoration models have been developed - cf. Refs. [5, 23]. On the other hand the total variation (TV) denoising scheme proposed by Rudin et al. [19], initiated the development of numerous PDE variational filtering techniques $[1,3,7,8,11,15,20,22]$.

\footnotetext{
*Corresponding author. Email addresses: tudor.barbu@iit.academiaromana-is.ro (T. Barbu), costica.morosanu@uaic.ro (C. Moroşanu)
} 
However, although such second-order PDE-based methods remove image blurring and preserve boundaries, they may cause another unintended problem - viz. staircase (or blocky) effect [6]. In contrast, nonlinear fourth-order diffusion-based models inspired by the influential You-Kaveh scheme [24], can successfully remove additive Gaussian noise and overcome the staircase effect - cf. [2,4]. Nevertheless, the over-filtering of the fourthorder diffusion models often affects the image and may produce undesired multiplicative speckle noise.

In this work, we develop a novel PDE-based technique, which successfully removes the additive noise, while avoiding or alleviating all the unintended effects mentioned. It is based on an improved second-order anisotropic diffusion model and a two-dimension Gaussian filter kernel. This model is discussed in Section 2 below. Section 3 deals with a fast-converging numerical approximation scheme based on the finite difference method in $[9,12]$ and consistent with the model under consideration. A rigorous mathematical analysis of this PDE-based model is provided in Section 4. In particular, we prove the existence and regularity of the classical solution for the corresponding nonlinear second-order diffusion-based scheme. In Section 5, we demonstrate the effectiveness of this restoration approach and compare it with other denoising models using the image quality measures [21]. Our conclusions are in Section 6.

\section{A Nonlinear Second-Order Anisotropic Diffusion Model}

We consider a novel second-order anisotropic diffusion-based model, which provides an effective detail-preserving image restoration. It is based on a boundary value problem for a nonlinear PDE — viz.

$$
\begin{aligned}
& \frac{\partial}{\partial t} u-\eta_{u}\left(\left\|\nabla G_{\sigma} * u\right\|\right) \nabla \cdot\left(\Psi^{u}(\|\nabla u\|) \nabla u\right)+\alpha\left(u-u_{0}\right)=0, \\
& u\left(0, x_{1}, x_{2}\right)=u_{0}\left(x_{1}, x_{2}\right), \quad\left(x_{1}, x_{2}\right) \in \Omega \subset R^{2} \\
& u\left(t, x_{1}, x_{2}\right)=0, \quad t \in[0, T], \quad \forall\left(x_{1}, x_{2}\right) \in \partial \Omega
\end{aligned}
$$

where $\alpha \in(0,1], u_{0}$ is the observed image, $G_{\sigma}$ the Gaussian kernel,

$$
G_{\sigma}\left(x_{1}, x_{2}\right)=\frac{1}{2 \pi \sigma^{2}} \exp \left(-\frac{x_{1}^{2}+x_{2}^{2}}{2 \sigma^{2}}\right)
$$

determined by the standard deviation parameter $\sigma>0$, and $\|\cdot\|$ refers to the $L^{2}$ norm.

The function $\eta_{u}:(0, \infty) \rightarrow(0, \infty)$ in the PDE-based model $(2.1)$ has the form

$$
\eta_{u}(s)=\frac{\left(\lambda s^{k}+v\right)^{1 /(k+1)}}{\xi},
$$

where $\lambda, v \in(0,4], \xi \geq 1.5, k \in(0,2]$. It is worth noting that the term $\eta_{u}\left(\left\|\nabla G_{\sigma} * u\right\|\right)$ controls the speed of this diffusion process and enhances the edges of the corresponding image. 
The edge-stopping (diffusivity) function $\Psi^{u}:[0, \infty) \rightarrow[0, \infty)$ in $(2.1)$ has the form

$$
\Psi^{u}(s)=\varepsilon\left(\frac{\delta(u)}{\beta \ln (\delta(u))+\gamma s_{1}^{2}}\right)^{1 / 3},
$$

where $\varepsilon \in(0,2), \gamma \in(1,5], \beta \in(0,1)$, and the conductance parameter $\delta$ is defined by

$$
\delta(u):=|r \mu(\|\nabla u\|)+\zeta \mathscr{M}(\|\nabla u\|)|, \quad r>0, \quad \zeta \in(0,1)
$$

with the respective averaging and median operators $\mu$ and $\mathscr{M}$.

The function (2.2) satisfies the main requirements for a successful restoration $[3,23]$ - e.g. it is positive, monotonically decreasing in $(0, \infty)$ and $\lim _{s \rightarrow \infty} \Psi^{u}(s)=0$.

Because of the presence of the term $\eta_{u}\left(\left\|\nabla G_{\sigma} * u\right\|\right)$, the nonlinear operator in the Eq. (2.1) does not represent the gradient of the energy functional. Therefore, the proposed second-order nonlinear diffusion-based scheme cannot be obtained from the minimisation of any energy cost functional, so that this scheme is not a variational PDE model.

The restored image is obtained from an observed image by solving the anisotropic diffusion model (2.1). The solution of this equation can be derived by the iterative algorithm introduced in the next section.

\section{Consistent Numerical Approximation Algorithm}

In this section we propose a robust numerical approximation scheme based on the finite difference method $[9,12]$ for the non-linear model $(2.1)$. More precisely, let $h$ and $\Delta t$ be, respectively, space and time grids and let

$$
x:=i h, \quad y:=j h, \quad t:=n \Delta t \quad \text { for all } i \in\{0, \cdots, I\}, \quad j \in\{0, \cdots, J\}, \quad n \in\{0, \cdots, N\}
$$

with the image frame $I h \times J h$.

The partial differential equation in (2.1) can be written in the form

$$
\frac{\partial}{\partial t} u=\eta_{u}\left(\left\|\nabla G_{\sigma} * u\right\|\right)\left(\frac{\partial}{\partial x_{1}}\left(\Psi^{u}(\|\nabla u\|) u_{x_{1}}\right)+\frac{\partial}{\partial x_{2}}\left(\Psi^{u}(\|\nabla u\|) u_{x_{2}}\right)\right)-\alpha\left(u-u_{0}\right)
$$

and discretised as follows. First, we compute the terms $\eta_{i, j}=\eta_{u}\left(\left\|\left(G_{\sigma} * u\right)_{i, j}\right\|\right)$ and $\Psi_{i, j}=$ $\Psi^{u}\left(\left\|u_{i, j}\right\|\right)$, approximating the gradient magnitude by central differences $[9,12]$ :

$$
\left\|u_{i, j}\right\|=\left(\left(\frac{u_{i+h, j}-u_{i-h, j}}{2 h}\right)^{2}+\left(\frac{u_{i, j+h}-u_{i, j-h}}{2 h}\right)^{2}\right)^{1 / 2}
$$

In addition, the terms

$$
\frac{\partial}{\partial x_{1}}\left(\Psi^{u}(\|\nabla u\|) u_{x_{1}}\right), \quad \frac{\partial}{\partial x_{2}}\left(\Psi^{u}(\|\nabla u\|) u_{x_{2}}\right)
$$


are, respectively, discretised as

$$
\begin{aligned}
& \Psi_{i+h / 2, j}\left(u_{i+h, j}-u_{i, j}\right)-\Psi_{i-h / 2, j}\left(u_{i, j}-u_{i-h, j}\right), \\
& \Psi_{i, j+h / 2}\left(u_{i, j+h}-u_{i, j}\right)-\Psi_{i, j-h / 2}\left(u_{i, j}-u_{i, j-h}\right),
\end{aligned}
$$

where

$$
\begin{aligned}
& \Psi_{i \pm h / 2, j}=\frac{\Psi_{i \pm h, j}+\Psi_{i, j}}{2}, \\
& \Psi_{i, j \pm h / 2}=\frac{\Psi_{i, j \pm h}+\Psi_{i, j}}{2} .
\end{aligned}
$$

The forward differences are then applied to the time derivative [12] and we arrive at the following implicit discretisation of the Eq. (3.1):

$$
\begin{aligned}
\frac{u_{i, j}^{n+\Delta t}-u_{i, j}^{n}}{\Delta t}= & \eta_{i, j}\left(\Psi_{i+h / 2, j}\left(u_{i+h, j}^{n}-u_{i, j}^{n}\right)-\Psi_{i-h / 2, j}\left(u_{i, j}^{n}-u_{i-h, j}^{n}\right)\right. \\
& \left.+\Psi_{i, j+h / 2}\left(u_{i, j+h}^{n}-u_{i, j}^{n}\right)-\Psi_{i, j-h / 2}\left(u_{i, j}^{n}-u_{i, j-h}^{n}\right)\right)-\alpha\left(u_{i, j}^{n}-u_{i, j}^{0}\right) .
\end{aligned}
$$

Using the parameters $\Delta t=1$ and $h=1$, we rewrite the implicit approximation algorithm (3.2) as an explicit numerical approximation scheme - viz.

$$
\begin{aligned}
u_{i, j}^{n+1}= & u_{i, j}^{n}\left[1-\alpha-\eta_{i, j}\left(\Psi_{i+1 / 2, j}+\Psi_{i-1 / 2, j}+\Psi_{i, j+1 / 2}+\Psi_{i, j-1 / 2}\right)\right] \\
& +u_{i+1, j}^{n} \eta_{i, j} \Psi_{i+1 / 2, j}+u_{i-1, j}^{n} \eta_{i, j} \Psi_{i-1 / 2, j} \\
& +u_{i, j+1}^{n} \eta_{i, j} \Psi_{i, j+1 / 2}+u_{i, j-1}^{n} \eta_{i, j} \Psi_{i, j-1 / 2}+\alpha u_{i, j}^{0}
\end{aligned}
$$

where $u_{0, j}^{n}=u_{1, j}^{n}, u_{I, j}^{n}=u_{I+1, j}^{n}, u_{i, 0}^{n}=u_{i, 1}^{n}$ and $u_{i, J}^{n}=u_{i, J+1}^{n}$. The iterative numerical approximation algorithm (3.3) is stable, consistent with nonlinear diffusion-based model (2.1) and converges fast to the exact solution representing the restored image.

\section{The Validity of the Diffusion Model}

In this section we study the validity of the above nonlinear model. In particular, we analyse the solvability of the model and the regularity of its solutions.

Let $k$ be a positive integer and $1 \leq p \leq \infty$. We denote by $W_{p}^{k, 2 k}(Q)$ the Sobolev space

$$
W_{p}^{k, 2 k}(Q):=\left\{y \in L^{p}(Q): \frac{\partial^{r}}{\partial t^{r}} \frac{\partial^{q}}{\partial x^{q}} y \in L^{p}(Q), \text { for } 2 r+q \leq k\right\},
$$

— cf. Ref. [13, p. 5], where $Q:=(0, T] \times \Omega$. Moreover, let $C^{1,2}(\bar{Q})\left(C^{1,2}(Q)\right)$ denote the set of all functions continuous in $\bar{Q}(Q)$ along with their derivatives $u_{t}, u_{x}, u_{x x}$ and $W_{\infty}^{2-2 / p}(\Omega)$, $W_{p}^{l, l / 2}(\Sigma)$ the corresponding Sobolev spaces with a non integral $l-c f .[13$, pp. 70,81$]$. 
Now we consider the problem (2.1) in a bounded domain $\Omega \subset \mathbb{R}^{2}$ with the boundary $\partial \Omega \in C^{2}$ for a finite time $T>0$, so that

$$
\begin{aligned}
& \frac{\partial}{\partial t} u\left(t, x_{1}, x_{2}\right)=\eta_{u}\left(\left\|\nabla G_{\sigma} * u\right\|\right) \operatorname{div}\left(\Psi^{u}(\|\nabla u\|) \nabla u\right) \\
& \quad-\alpha\left(u\left(t, x_{1}, x_{2}\right)-u_{0}\left(x_{1}, x_{2}\right)\right)+f\left(t, x_{1}, x_{2}\right) \text { in } Q, \\
& \frac{\partial}{\partial v} u\left(t, x_{1}, x_{2}\right)=0 \quad \text { on } \Sigma, \\
& u\left(0, x_{1}, x_{2}\right)=u_{0}\left(x_{1}, x_{2}\right) \quad \text { on } \Omega,
\end{aligned}
$$

where $\Sigma:=(0, T] \times \partial \Omega, u_{0}\left(x_{1}, x_{2}\right) \in W_{\infty}^{2-2 / p}(\Omega), p \geq 2$ and $\partial u_{0}\left(x_{1}, x_{2}\right) / \partial v=0$. The other terms in (4.1) are as before.

Definition 4.1. Any solution of the problem (4.1) is called the classical solution if it is continuous in $\bar{Q}$ and has continuous derivatives $u_{t}, u_{x}, u_{x x}$ in $Q$.

For the sake of convenience, we rewrite the problem (4.1) in the following equivalent form

$$
\begin{aligned}
& \frac{\partial}{\partial t} u\left(t, x_{1}, x_{2}\right)-\eta_{u}\left(\left\|\nabla G_{\sigma} * u\right\|\right) \frac{\partial}{\partial u_{x_{j}}}\left(\Psi^{u}(\|\nabla u\|) u_{x_{i}}\right) u_{x_{j} x_{i}} \\
& \quad+A\left(t, x_{1}, x_{2}, u, u_{x_{i}}\right)=\alpha u_{0}\left(x_{1}, x_{2}\right)+f\left(t, x_{1}, x_{2}\right) \text { in } Q, \\
& \frac{\partial}{\partial v} u\left(t, x_{1}, x_{2}\right)=0 \quad \text { on } \Sigma, \\
& u\left(0, x_{1}, x_{2}\right)=u_{0}\left(x_{1}, x_{2}\right) \quad \text { on } \Omega,
\end{aligned}
$$

where

$$
\begin{aligned}
A\left(t, x_{1}, x_{2}, u, u_{x_{i}}\right)= & -\eta_{u}\left(\left\|\nabla G_{\sigma} * u\right\|\right)\left[\frac{\partial}{\partial u}\left(\Psi^{u}(\|\nabla u\|) u_{x_{i}}\right) u_{x_{i}}+\frac{\partial}{\partial x_{i}}\left(\Psi^{u}(\|\nabla u\|) u_{x_{i}}\right)\right] \\
& +\alpha u\left(t, x_{1}, x_{2}\right)
\end{aligned}
$$

and

$$
u_{x_{i}}:=\frac{\partial}{\partial x_{i}} u\left(t, x_{1}, x_{2}\right), \quad u_{x_{j} x_{i}}:=\frac{\partial^{2}}{\partial x_{j} \partial x_{i}} u\left(t, x_{1}, x_{2}\right), \quad i, j=1,2 .
$$

Our study of the solvability of the problem (4.2) in the space $W_{p}^{1,2}(Q)$ is based on the LeraySchauder degree theory and the $L^{p}$-theory of linear and quasi-linear parabolic equations [13]. More precisely, the following theorem is true.

Theorem 4.1. Let $u\left(t, x_{1}, x_{2}\right) \in C^{1,2}(Q)$ be a classical solution of Eq. (4.2) such that

$$
\frac{\partial}{\partial v} u\left(t, x_{1}, x_{2}\right)=0
$$

on the lateral surface $\Sigma$ of the cylinder $Q$ and for positive numbers $M, M_{1}, M_{2}$ and $M_{3}$ one has 
$\mathrm{I}_{1}$. If $\left(t, x_{1}, x_{2}\right) \in Q$, then $\left|u\left(t, x_{1}, x_{2}\right)\right|<M$ and for arbitrary $q$, the function $\Psi^{u}(\|\nabla u\|) q$ is continuous, differentiable with respect to $x=\left(x_{1}, x_{2}\right)$, $u$ and $q$ and satisfies the condition of uniform parabolicity - i.e.

$$
v|y|^{2} \leq \frac{\partial}{\partial q_{j}}\left(\Psi^{u}(\|\nabla u\|) q\right) y_{i} y_{j} \leq \mu|y|^{2}, \quad v>0, \quad y \in \mathbb{R}^{n}
$$

and

$$
\begin{aligned}
& {\left[\left|\Psi^{u}(\|\nabla u\|) u_{x_{i}}\right|+\left|\frac{\partial}{\partial u}\left(\Psi^{u}(\|\nabla u\|) u_{x_{i}}\right)\right|\right](1+|q|)} \\
& +\left|\frac{\partial}{\partial x_{1}}\left(\Psi^{u}(\|\nabla u\|) u_{x_{1}}\right)\right|+\left|\frac{\partial}{\partial x_{2}}\left(\Psi^{u}(\|\nabla u\|) u_{x_{1}}\right)\right| \\
& +\left|\frac{\partial}{\partial x_{1}}\left(\Psi^{u}(\|\nabla u\|) u_{x_{2}}\right)\right|+\left|\frac{\partial}{\partial x_{2}}\left(\Psi^{u}(\|\nabla u\|)\right) u_{x_{2}}\right| \\
& +\left|u\left(t, x_{1}, x_{2}\right)\right| \leq \mu(1+|q|)^{2} .
\end{aligned}
$$

$\mathrm{I}_{2}$. For any sufficiently small $\varepsilon>0$, the functions $\eta_{u}$, $u$ and $\Psi^{u}$ satisfy the inequalities

$$
\eta_{u}\left(\left\|\nabla G_{\sigma} * u\right\|\right)<M_{1}, \quad\|u\|_{L^{s}(Q)} \leq M_{3}, \quad\left\|\Psi^{u}(\|\nabla u\|) u_{x_{i}}\right\|_{L^{r}(Q)}<M_{2}, \quad i=1,2,
$$

where

$$
r=\left\{\begin{array}{ll}
\max \{p, 4\}, & p \neq 4, \\
4+\varepsilon, & p=4,
\end{array} \text { and } s= \begin{cases}\max \{p, 2\}, & p \neq 2 \\
2+\varepsilon, & p=2\end{cases}\right.
$$

Then, for any $f \in L^{p}(Q)$ and $u_{0} \in W_{\infty}^{2-2 / p}(\Omega), p \neq 3 / 2$, the problem (4.2) has a solution $u \in W_{p}^{1,2}(Q)$, such that

$$
\|u\|_{W_{p}^{1,2}(Q)} \leq C\left(\left\|u_{0}\right\|_{W_{\infty}^{2-2 / p}(\Omega)}+\|f\|_{L^{p}(Q)}\right),
$$

where the constant $C>0$ is independent of $u$ and $f$.

Proof. To prove this theorem, we use the Leray-Schauder principle. Consider the Banach space $B=W_{p}^{0,1}(Q)$ endowed with the norm $\|v\|_{B}=\|v\|_{L^{p}(Q)}+\left\|v_{x}\right\|_{L^{p}(Q)}$, and a nonlinear operator $H$ defined by

$$
H(v, \lambda):=u(v, \lambda) \text { for all }(v, \lambda) \in W_{p}^{0,1}(Q) \times[0,1]
$$

where $u(v, \lambda)$ is the unique solution to the following linear boundary value problem

$$
\begin{aligned}
& \frac{\partial}{\partial t} u\left(t, x_{1}, x_{2}\right)-\left[\lambda \eta_{v}\left(\left\|\nabla G_{\sigma} * v\right\|\right) \frac{\partial}{\partial v_{x_{j}}}\left(\Psi^{v}(\|\nabla v\|) v_{x_{i}}\right)+(1-\lambda) \delta_{i}^{j}\right] \\
& \quad \times u_{x_{i} x_{j}}=-\lambda\left[A\left(t, x_{1}, x_{2}, v, v_{x_{i}}\right)-\alpha u_{0}\left(x_{1}, x_{2}\right)+f\left(t, x_{1}, x_{2}\right)\right] \text { in } Q, \\
& \frac{\partial}{\partial v} u\left(t, x_{1}, x_{2}\right)=0 \quad \text { on } \Sigma, \\
& u\left(0, x_{1}, x_{2}\right)=\lambda u_{0}\left(x_{1}, x_{2}\right) \quad \text { on } \Omega .
\end{aligned}
$$

Our theorem will be proved if we show that the nonlinear operator $H$ has two properties: 
A. The operator $H$ is well-defined.

B. The operator $H$ is continuous and compact.

We start with the definition of the operator $H$.

A. The operator $H$ is well-defined if the problem (4.6) has a unique solution. The Eq. (4.6) shows that if $v \in W_{p}^{0,1}(Q)$, then $A\left(t, x_{1}, x_{2}, v, v_{x_{i}}\right)+f\left(t, x_{1}, x_{2}\right) \in L^{p}(Q)$ and according to [13, p. 341-342], the problem (4.6) has unique solution $u$ such that

$$
u=u(v, \lambda) \in W_{p}^{1,2}(Q), \quad \forall v \in W_{p}^{0,1}(Q), \quad \forall \lambda \in[0,1] .
$$

Taking into account that $W_{p}^{1,2}(Q) \subset W_{p}^{0,1}(Q)-$ cf. [14, p. 24], we obtain that $H(v, \lambda)=$ $u \in W_{p}^{0,1}(Q)$ for all $v \in W_{p}^{0,1}(Q)$ and $\lambda \in[0,1]$.

B. Let us now show that $H$ is continuous and compact. Let $v^{n} \rightarrow v$ in $W_{p}^{0,1}(Q)$ and $\lambda_{n} \rightarrow \lambda$ in $[0,1]$. Using the notation

$$
u^{n, \lambda_{n}}=H\left(v^{n}, \lambda_{n}\right), \quad u^{n, \lambda}=H\left(v^{n}, \lambda\right) \quad \text { and } \quad u^{\lambda}=H(v, \lambda)
$$

and considering the difference $H\left(v^{n}, \lambda_{n}\right)-H\left(v^{n}, \lambda\right)$, we obtain from the Eqs. (4.5) and (4.6) that

$$
\begin{aligned}
& \frac{\partial}{\partial t}\left(u^{n, \lambda_{n}}-u^{n, \lambda}\right)-\left[\lambda \eta_{v^{n}}\left(\left\|\nabla G_{\sigma} * v^{n}\right\|\right) \frac{\partial}{\partial v_{x_{j}}^{n}}\left(\Psi^{v^{n}}\left(\left\|\nabla v^{n}\right\|\right) v_{x_{i}}^{n}\right)+(1-\lambda) \delta_{i}^{j}\right] \\
& \quad \times\left(u_{x_{i} x_{j}}^{n, \lambda_{n}}-u_{x_{i} x_{j}}^{n, \lambda}\right)=-\left(\lambda_{n}-\lambda\right)\left\{\left[\eta_{v^{n}}\left(\left\|\nabla G_{\sigma} * v^{n}\right\|\right) \frac{\partial}{\partial v_{x_{j}}^{n}}\left(\Psi^{v^{n}}\left(\left\|\nabla v^{n}\right\|\right) v_{x_{i}}^{n}\right)-\delta_{i}^{j}\right] u_{x_{i} x_{j}}^{n, \lambda_{n}}\right. \\
& \left.\quad+A\left(t, x, v^{n}, v_{x_{i}}^{n}\right)-\alpha u_{0}\left(x_{1}, x_{2}\right)+f\left(t, x_{1}, x_{2}\right)\right\} \quad \text { in } Q \\
& \frac{\partial}{\partial v}\left(u^{n, \lambda_{n}}-u^{n, \lambda}\right)=0 \quad \text { on } \Sigma, \\
& \left(u^{n, \lambda_{n}}-u^{n, \lambda}\right)\left(0, x_{1}, x_{2}\right)=\left(\lambda_{n}-\lambda\right) u_{0}\left(x_{1}, x_{2}\right) \text { in } \Omega .
\end{aligned}
$$

The right-hand side in (4.7) belongs to $L^{p}(Q)$, since $u^{n, \lambda_{n}} \in W_{p}^{1,2}(Q)$. Therefore, the $L^{p}$ theory of PDE yields the estimate

$$
\begin{aligned}
\left\|u^{n, \lambda_{n}}-u^{n, \lambda}\right\|_{W_{p}^{1,2}(Q)} \leq & C\left|\lambda_{n}-\lambda\right|\left(\left\|\left(\eta_{v^{n}}\left(\left\|\nabla G_{\sigma} * v^{n}\right\|\right) \frac{\partial}{\partial v_{x_{j}}^{n}}\left(\Psi^{v^{n}}\left(\left\|\nabla v^{n}\right\|\right) v_{x_{i}}^{n}\right)-\delta_{i}^{j}\right) u_{x_{i} x_{j}}^{n, \lambda_{n}}\right\|_{L^{p}(Q)}\right. \\
& \left.+\left\|A\left(t, x, v^{n}, v_{x_{i}}^{n}\right)\right\|_{L^{p}(Q)}+\left\|u_{0}\right\|_{W_{\infty}^{2-2 / p}(\Omega)}+\|f\|_{L^{p}(Q)}\right)
\end{aligned}
$$

with a constant $C\left(|\Omega|, p, \alpha, M, M_{1}, M_{2}, M_{3}\right)$.

The inequality (4.3), condition $\mathrm{I}_{2}$ and the inclusion $u_{x_{i} x_{j}}^{n, \lambda_{n}} \in L^{p}(Q)$ imply the boundedness of $A\left(t, x, v^{n}, v_{x_{i}}^{n}\right),\left(\eta_{v^{n}}\left(\left\|\nabla G_{\sigma} * v^{n}\right\|\right) \frac{\partial}{\partial v_{x_{j}}^{n}}\left(\Psi^{v^{n}}\left(\left\|\nabla v^{n}\right\|\right) v_{x_{i}}^{n}\right)-\delta_{i}^{j}\right) u_{x_{i} x_{j}}^{n, \lambda_{n}}, u_{0}$ and $f$ in $L^{p}(Q)$, and since $\lambda_{n} \rightarrow \lambda$, we obtain

$$
\left\|u^{n, \lambda_{n}}-u^{n, \lambda}\right\|_{W_{p}^{1,2}(Q)} \rightarrow 0 \text { for } n \rightarrow \infty .
$$


In order to evaluate the difference $H\left(v^{n}, \lambda\right)-H(v, \lambda)$, we use (4.5) and (4.6), so that

$$
\begin{aligned}
& \frac{\partial}{\partial t}\left(u^{n, \lambda}-u^{\lambda}\right)-\left[\lambda \eta_{v^{n}}\left(\left\|\nabla G_{\sigma} * v^{n}\right\|\right) \frac{\partial}{\partial v_{x_{j}}^{n}}\left(\Psi^{v^{n}}\left(\left\|\nabla v^{n}\right\|\right) v_{x_{i}}^{n}\right)+(1-\lambda) \delta_{i}^{j}\right] \\
& \quad \times\left(u_{x_{\lambda} x_{j}}^{n, \lambda}-u_{x_{i} x_{j}}^{\lambda}\right)=-\lambda\left[\eta_{v^{n}}\left(\left\|\nabla G_{\sigma} * v^{n}\right\|\right) \frac{\partial}{\partial v_{x_{j}}^{n}}\left(\Psi^{v^{n}}\left(\left\|\nabla v^{n}\right\|\right) v_{x_{i}}^{n}\right)\right. \\
& \left.\quad-\eta_{v}\left(\left\|\nabla G_{\sigma} * v\right\|\right) \frac{\partial}{\partial v_{x_{j}}}\left(\Psi^{v}(\|\nabla v\|) v_{x_{i}}\right)\right] u_{x_{i} x^{j}}^{\lambda} \\
& \quad-\lambda\left[A\left(t, x, v^{n}, v_{x_{i}}^{n}-A\left(t, x, v, v_{x_{i}}\right)\right] \text { in } Q,\right. \\
& \frac{\partial}{\partial v}\left(u^{n, \lambda}-u^{\lambda}\right)=0 \text { on } \Sigma, \\
& \left(u^{n, \lambda}-u^{\lambda}\right)\left(0, x_{1}, x_{2}\right)=0 \text { on } \Omega .
\end{aligned}
$$

Using the $L^{p}$-theory of PDE again, we arrive at the estimate

$$
\begin{aligned}
\left\|u^{n, \lambda}-u^{\lambda}\right\|_{W_{p}^{1,2}(Q)} \leq & C\left[\|\left(\eta_{v^{n}}\left(\left\|\nabla G_{\sigma} * v^{n}\right\|\right) \frac{\partial}{\partial v_{x_{j}}^{n}}\left(\Psi^{v^{n}}\left(\left\|\nabla v^{n}\right\|\right) v_{x_{i}}^{n}\right)\right.\right. \\
& \left.-\eta_{v}\left(\left\|\nabla G_{\sigma} * v\right\|\right) \frac{\partial}{\partial v_{x_{j}}}\left(\Psi^{v}(\|\nabla v\|) v_{x_{i}}\right)\right) u_{x_{i} x_{j}}^{\lambda} \|_{L^{p}(Q)} \\
& \left.+\left\|A\left(t, x, v^{n}, v_{x_{i}}^{n}\right)-A\left(t, x, v, v_{x_{i}}\right)\right\|_{L^{p}(Q)}\right]
\end{aligned}
$$

with a constant $C$. Since all terms in the right-hand side of this inequality are bounded and $v^{n}$ converges to $v$ in $W_{p}^{0,1}(Q)$, it follows that

$$
\left\|u^{n, \lambda}-u^{\lambda}\right\|_{W_{p}^{1,2}(Q)} \rightarrow 0 \quad \text { as } \quad n \rightarrow \infty
$$

Making use of the relations (4.8) and (4.10), we show the continuity of the nonlinear operator $H$. Moreover, the mapping $H$ defined by (4.5) is compact, what can easily be seen by writing it as the composition

$$
B=W_{p}^{0,1}(Q) \times[0,1] \rightarrow W_{p}^{1,2}(Q) \hookrightarrow B=W_{p}^{0,1}(Q),
$$

where the second map is an compact inclusion due to Lions-Peeter embedding theorem cf. Ref. [14, p. 21].

Now we establish the existence of a number $\delta>0$ such that

$$
(u, \lambda) \in W_{p}^{0,1}(Q) \times[0,1] \quad \text { with } \quad u=H(u, \lambda) \Rightarrow\|u\|_{B}<\delta .
$$


The equality $u=H(u, \lambda)$ in (4.11) is equivalent to

$$
\begin{aligned}
& \frac{\partial}{\partial t} u\left(t, x_{1}, x_{2}\right)-\left[\lambda \eta_{u}\left(\left\|\nabla G_{\sigma} * u\right\|\right) \frac{\partial}{\partial u_{x_{j}}}\left(\Psi^{u}(\|\nabla u\|) u_{x_{i}}\right)+(1-\lambda) \delta_{i}^{j}\right] \\
& \quad \times u_{x_{i} x_{j}}=-\lambda\left[A\left(t, x_{1}, x_{2}, u, u_{x_{i}}\right)-\alpha u_{0}\left(x_{1}, x_{2}\right)+f\left(t, x_{1}, x_{2}\right)\right] \text { in } Q, \\
& \frac{\partial}{\partial v} u\left(t, x_{1}, x_{2}\right)=0 \quad \text { on } \Sigma, \\
& u\left(0, x_{1}, x_{2}\right)=\lambda u_{0}\left(x_{1}, x_{2}\right) \text { on } \Omega .
\end{aligned}
$$

Taking into account the $L^{p}$-theory of PDFs, assumptions (4.3) and $I_{2}$, for $p \neq 3 / 2$ we conclude that

$$
\|u\|_{W_{p}^{1,2}(Q)} \leq C\left(\left\|u_{0}\right\|_{W_{\infty}^{2-2 / p}(\Omega)}+\|f\|_{L^{p}(Q)}\right)
$$

with a constant $C\left(|\Omega|, p, \alpha, v, M, M_{1}, M_{2}, M_{3}\right)>0$. This inequality and the embedding $W_{p}^{1,2}(Q) \subset W_{p}^{0,1}(Q)$ yield

$$
\|u\|_{W_{p}^{0,1}(Q)} \leq C\|u\|_{W_{p}^{1,2}(Q)},
$$

thus confirming the validity of (4.11).

Considering the ball

$$
B_{\delta}:=\left\{u \in B:\|u\|_{B}<\delta\right\}
$$

we note that the inequality (4.11) implies that $H(u, \lambda) \neq u$ for any $u \in \partial B_{\delta}$ and $\lambda \in[0,1]$, provided that $\delta>0$ is sufficiently large. Moreover, following the arguments of [16] and [17], we conclude that the problem (4.2) has a solution $u \in W_{p}^{1,2}(Q)$, and the inequality (4.13) leads to the estimate (4.4).

Remark 4.1. The nonlinear operator $H$ in (4.5) depends on $\lambda \in[0,1]$ and for $\lambda=1$ its fixed points are the solutions of (4.2)

\section{Restoration Experiments and Method Comparison}

The proposed diffusion-based restoration approach has been successfully tested on the images corrupted by white additive Gaussian noise. The images were acquired from the USC - SIPI database.

The performance of this technique has been assessed by the Peak Signal to Noise Ratio (PSNR), the Signal to Noise Ratio (SNR) and Mean-squared Error (MSE) — cf. Ref. [17]. The following set of the model parameters

$$
\begin{array}{llll}
\alpha=0.6, & \lambda=1.3, & v=1.5, \quad k=0.35, \quad \xi=1.66, \quad \varepsilon=0.5, \\
\gamma=3.7, \quad \beta=0.4, & \quad r=0.6, \quad \zeta=0.25, \quad N=12
\end{array}
$$

ensures optimal image restoration. It was empirically identified by the trial and error method. It is worth noting that the method successfully removes additive noise, overcomes blurring effect, reduces the image staircasing and does not generate multiplicative noise, 
Table 1: Average PSNR and SSIM for several filtering models.

\begin{tabular}{||l|c|c||}
\hline Image Filtering technique & Peak Signal to Noise Ratio & Structural Similarity Index \\
\hline The proposed AD restoration & $29.6128(\mathrm{~dB})$ & 0.8751 \\
\hline Gaussian 2D filter & $23.1857(\mathrm{~dB})$ & 0.4532 \\
\hline Average filter & $24.9836(\mathrm{~dB})$ & 0.5174 \\
\hline Wiener 2D filter & $25.7205(\mathrm{~dB})$ & 0.8271 \\
\hline Perona-Malik 1 & $25.4753(\mathrm{~dB})$ & 0.6789 \\
\hline Perona-Malik 2 & $26.1462(\mathrm{~dB})$ & 0.7514 \\
\hline ROF - TV Denoising & $27.8543(\mathrm{~dB})$ & 0.8356 \\
\hline
\end{tabular}

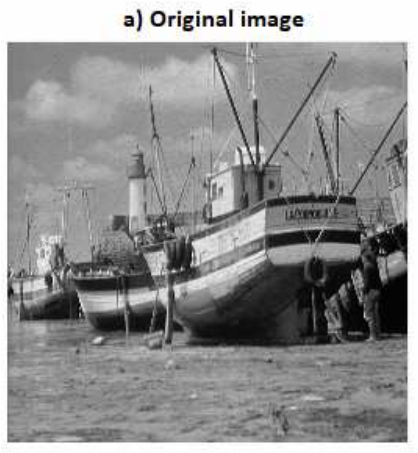

d) Gaussian filter

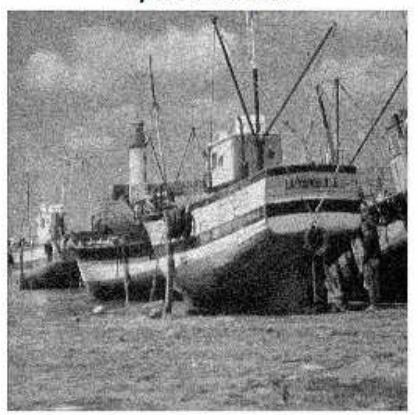

g) Perona-Malik 1

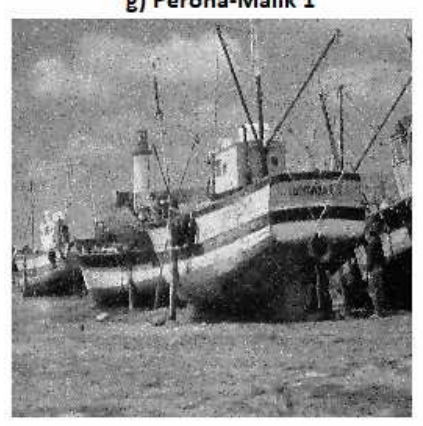

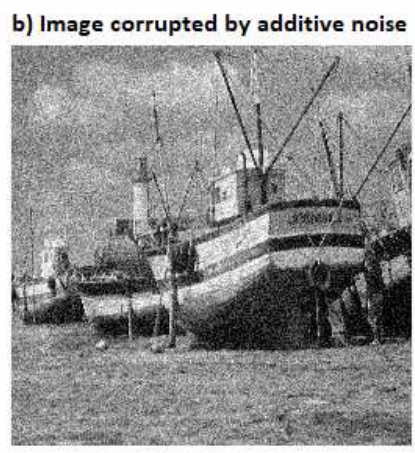

e) Average filter

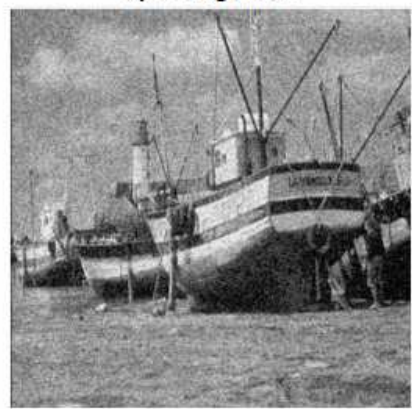

h) Perona-Malik 2

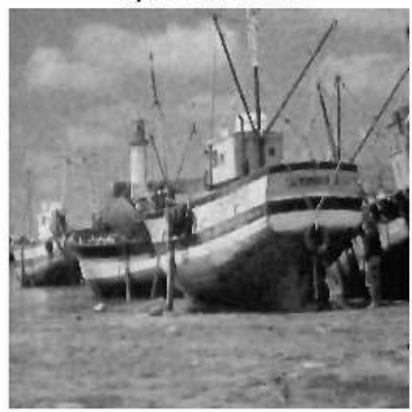

c) Proposed AD restoration

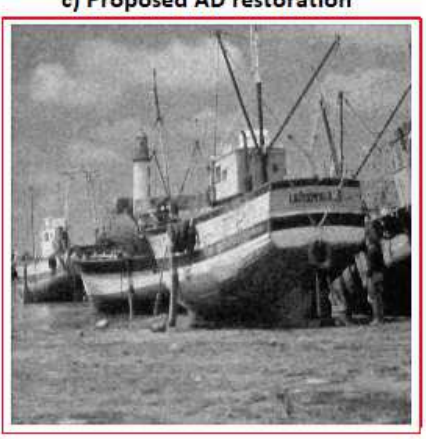

f) Wiener filtering

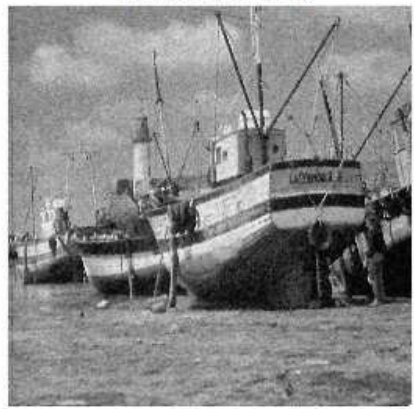

i) TV Denoising

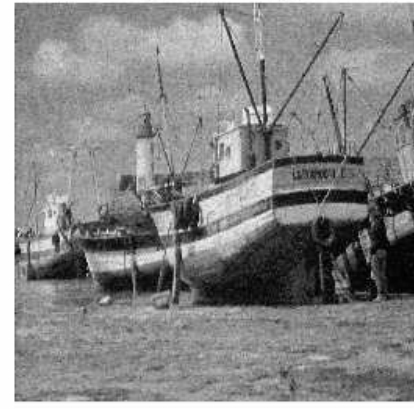

Figure 1: The restoration of the Boat image. 
thus preserving boundaries and all the essential image features very well. Moreover, since the iterative numerical algorithm for the corresponding PDE converges fast to the optimal restoration, the method requires a low execution time, which depends on the amount of noise present.

Comparing this method with the existing PDE and non-PDE restoration models, we observe that it clearly outperforms the conventional two-dimension image filters [10] such as average, Gaussian $2 D$ and Wiener, and also the linear PDE-based filters, providing a better denoising and avoiding the undesired blurring effect. It also outperforms the nonlinear second-order diffusion-based and variational schemes motivated by Perona-Malik and ROF-TV denoising models [5, 19,23], which encounter difficulties with staircase effect. Moreover, the solutions obtained by this method are better than even those found by the fourth-order You-Kaveh alike PDE models [24], since there is no multiplicative (speckle) noise, the edgesand, the other image details are better preserved and the execution is much faster.

Table 1 shows the average PSNR and SSIM (Structural Similarity Index) for various denoising methods, with the introduced anisotropic diffusion (AD) approach achieving the best results. Note that the other methods data are taken of [21].

Fig. 1 demonstrates one of numerous filtering simulations for the Boat image. The original image from USC-SIPI database, is corrupted by the Gaussian additive noise characterised by $\mu=0.11$ and the variance $0.02-$ cf. Fig. 1(b). The restoration results for various methods are presented in Figs. 1(c)-1(i).

\section{Conclusions}

We propose a novel second-order nonlinear anisotropic diffusion-based model for Gaussian additive noise removal. The method is based on a properly constructed edge-stopping function and provides an efficient detail-preserving denoising. The corresponding PDE model is solved by a robust finite-difference based iterative approximation scheme consistent with the diffusion model. The method converges very fast to the model solution, the existence and regularity of which is rigorously proved.

The proposed restoration method successfully removes additive noise, overcomes blurring effect, reduces the image staircasing and does not generate multiplicative noise, thus preserving boundaries and all the essential image features very well. It outperforms many other approaches and can be used in edge detection and object detection models and in image inpainting problems.

\section{References}

[1] T. Barbu, Variational Image Denoising Approach with Diffusion Porous Media Flow, Abstr. Appl. Anal. 2013, 856876 (2013).

[2] T. Barbu, Nonlinear Fourth-order Diffusion-based Model for Image Denoising, Soft Computing Applications, Adv. Intell. Syst. Comput. 633, Balas V., Jain L., Balas M. (Eds), Springer (2017). 
[3] T. Barbu and A. Favini, Rigorous mathematical investigation of a nonlinear anisotropic diffusionbased image restoration model, Electron. J. Dfferential Equations Equations 129, 1-9 (2014).

[4] T. Barbu, I. Munteanu, A Nonlinear Fourth-order Diffusion-based Model for Image Denoising and Restoration, Proc. Rom. Acad. Ser. A Math. Phys. Tech. Sci. Inf. Sci. 18, 108-115 (2017).

[5] M. Black, G. Shapiro, D. Marimont and D. Heeger, Robust anisotropic diffusion, IEEE Trans. Image Processing 7, 421-432 (1998).

[6] A. Buades, B. Coll and J. Morel, The staircasing effect in neighborhood filters and its solution, IEEE Trans. Image Process. 15, no. 6, 1499-1505 (2006).

[7] J. Cai, S. Osher and Z. Shen, Split Bregman methods and frame based image restoration, Multiscale Model. Sim. 8, 337-369 (2009).

[8] Q. Chen, P. Montesinos, Q. Sun, P. Heng and D. Xia, Adaptive total variation denoising based on difference curvature, Image Vis. Comput. 28, 298-306 (2010).

[9] D. Gleich, Finite Calculus: A Tutorial for Solving Nasty Sums, Stanford University (2005).

[10] R. Gonzalez and R. Woods, Digital Image Processing, Prentice Hall (2001).

[11] Y. Hu and M. Jacob, Higher degree total variation (HDTV) regularization for image recovery, IEEE Trans. Image Process. 21, 2559-2571 (2012).

[12] P. Johnson, Finite Difference for PDEs, University of Manchester (2008).

[13] O.A. Ladyženskaya, B.A. Solonnikov and N.N. Ural'ceva, Linear and Quasi-linear Equations of Parabolic Type, AMS (1968).

[14] J.L. Lions, Control of Distributed Singular Systems, Gauthier-Villars (1985).

[15] L. Ma, T. Zeng and G. Li, Hybrid variational model for texture image restoration, East. Asia. J. Appl. Math. 7, 629-642 (2017).

[16] C. Moroşanu, Analysis and optimal control of phase-field transition system: Fractional steps methods, Bentham Science Publishers (2012).

[17] C. Moroşanu, Well-posedness for a phase-field transition system endowed with a polynomial nonlinearity and a general class of nonlinear dynamic boundary conditions, J. Fixed Point Theory Appl. 18, 225-250 (2016).

[18] P. Perona and J. Malik, Scale-space and edge detection using anisotropic diffusion, in: Proc. of IEEE Computer Society Workshop on Computer Vision, pp. 16-22, Nov. 1987.

[19] L. Rudin, S. Osher and E. Fatemi, Nonlinear total variation based noise removal algorithms, Physica D: Nonlinear Phenomena 60, 259-268 (1992).

[20] F. Sciacchitano, Y. Dong and M.S. Andersen Total variation based parameter-free model for impulse noise removal, Numer. Math. Theor. Meth. Appl. 10, 186-204 (2017).

[21] K.H. Thung and P. Raveendran, A survey of image quality measures, in: Proc. of the International Conference for Technical Postgraduates (TECHPOS), pp. 1-4, Kuala Lumpur (2009).

[22] T. Wei, L. Wang, P. Lin, J. Chen, Y. Wang and H. Zheng Learning non-negativity constrained variation for imaged denoising and deblurring, Numer. Math. Theor. Meth. Appl. 10, 852-871 (2017).

[23] J. Weickert, Anisotropic Diffusion in Image Processing, European Consortium for Mathematics in Industry, B.G. Teubner (1998).

[24] Y.L. You and M. Kaveh, Fourth-order partial differential equations for noise removal, IEEE Trans. Image Process. 9, 1723-1730 (2000). 